\title{
The Racah Algebra as a Subalgebra of the Bannai-Ito Algebra
}

\author{
Hau-Wen HUANG
}

Department of Mathematics, National Central University, Chung-Li 32001, Taiwan

E-mail: hauwenh@math.ncu.edu.tw

Received May 22, 2020, in final form July 31, 2020; Published online August 10, 2020

https://doi.org/10.3842/SIGMA.2020.075

\begin{abstract}
Assume that $\mathbb{F}$ is a field with char $\mathbb{F} \neq 2$. The Racah algebra $\Re$ is a unital associative $\mathbb{F}$-algebra defined by generators and relations. The generators are $A, B, C, D$ and the relations assert that $[A, B]=[B, C]=[C, A]=2 D$ and each of $[A, D]+A C-B A$, $[B, D]+B A-C B,[C, D]+C B-A C$ is central in $\Re$. The Bannai-Ito algebra $\mathfrak{B} \mathfrak{I}$ is a unital associative $\mathbb{F}$-algebra generated by $X, Y, Z$ and the relations assert that each of $\{X, Y\}-Z,\{Y, Z\}-X,\{Z, X\}-Y$ is central in $\mathfrak{B} \mathfrak{I}$. It was discovered that there exists an $\mathbb{F}$-algebra homomorphism $\zeta: \Re \rightarrow \mathfrak{B I}$ that sends $A \mapsto \frac{(2 X-3)(2 X+1)}{16}, B \mapsto \frac{(2 Y-3)(2 Y+1)}{16}$, $C \mapsto \frac{(2 Z-3)(2 Z+1)}{16}$. We show that $\zeta$ is injective and therefore $\Re$ can be considered as an $\mathbb{F}$-subalgebra of $\mathfrak{B I}$. Moreover we show that any Casimir element of $\Re$ can be uniquely expressed as a polynomial in $\{X, Y\}-Z,\{Y, Z\}-X,\{Z, X\}-Y$ and $X+Y+Z$ with coefficients in $\mathbb{F}$.
\end{abstract}

Key words: Bannai-Ito algebra; Racah algebra; Casimir elements

2020 Mathematics Subject Classification: 81R10; 81R12

\section{Introduction}

Throughout this paper we adopt the following conventions: Assume that $\mathbb{F}$ is a field with char $\mathbb{F} \neq 2$. Let $\mathbb{N}$ denote the set of all nonnegative integers. The bracket $[$,$] stands for the$ commutator and the curly bracket $\{$,$\} stands for the anticommutator. An algebra is meant to$ be an associative algebra with unit 1 and a subalgebra is a subset of the parent algebra which is closed under the operations and has the same unit.

The Racah algebra $[19,23]$ and the Bannai-Ito algebra [26] are the $\mathbb{F}$-algebras defined by generators and relations to give the algebraic interpretations of the Racah polynomials and the Bannai-Ito polynomials, respectively. At first, the description of those relations involved several parameters. In recent papers $[7,9,16,18]$ the role of the parameters is replaced by the central elements. The contemporary Racah and Bannai-Ito algebras are defined as follows: The Racah algebra $\Re$ is an $\mathbb{F}$-algebra generated by $A, B, C, D$ and the relations assert that

$$
[A, B]=[B, C]=[C, A]=2 D
$$

and each of

$$
\alpha=[A, D]+A C-B A, \quad \beta=[B, D]+B A-C B, \quad \gamma=[C, D]+C B-A C
$$

is central in $\Re$. Note that

$$
\delta=A+B+C
$$

is also central in $\Re$. The Bannai-Ito algebra $\mathfrak{B} \mathfrak{I}$ is an $\mathbb{F}$-algebra generated by $X, Y, Z$ and the relations assert that each of

$$
\kappa=\{X, Y\}-Z, \quad \lambda=\{Y, Z\}-X, \quad \mu=\{Z, X\}-Y
$$


is central in $\mathfrak{B} \mathfrak{I}$. The applications to the Racah problems for $\mathfrak{s u}(2), \mathfrak{s u}(1,1), \mathfrak{s l}_{-1}(2)$ and the connections to the Laplace-Dunkl and Dirac-Dunkl equations on the 2-sphere have been explored in $[3,6,8,11,12,13,14,15,17,19,20,23]$. For more information and recent progress, see $[2,4,5,7,9,10,22]$.

A result of [16] made the following link between the Racah algebra $\Re$ and the Bannai-Ito algebra $\mathfrak{B} \mathfrak{I}$. The standard realization for $\mathfrak{B} \mathfrak{I}$ is a representation $\pi: \mathfrak{B I} \rightarrow \operatorname{End}(\mathbb{F}[x])$ given in [26, Section 4]. Inspired by $\pi$, a representation $\tau: \Re \rightarrow \operatorname{End}(\mathbb{F}[x])$ was constructed in $[16$, Section 2] as well as an $\mathbb{F}$-algebra homomorphism $\zeta: \Re \rightarrow \mathfrak{B I}$ that sends

$$
A \mapsto \frac{(2 X-3)(2 X+1)}{16}, \quad B \mapsto \frac{(2 Y-3)(2 Y+1)}{16}, \quad C \mapsto \frac{(2 Z-3)(2 Z+1)}{16} .
$$

Briefly $\tau$ is the composition of $\zeta$ followed by $\pi$. The main result of this paper is to prove that $\zeta$ is injective. To see this we derive the following results. We show that the monomials

$$
A^{i} B^{j} C^{k} D^{\ell} \alpha^{r} \beta^{s} \quad \text { for all } i, j, k, \ell, r, s \in \mathbb{N}
$$

are an $\mathbb{F}$-basis for $\Re$ and the monomials

$$
X^{i} Y^{j} Z^{k} \kappa^{r} \lambda^{s} \mu^{t} \quad \text { for all } i, j, k, r, s, t \in \mathbb{N}
$$

are an $\mathbb{F}$-basis for $\mathfrak{B I}$. We consider the following $\mathbb{F}$-subspaces of $\mathfrak{B} \mathfrak{I}$ induced from the basis (1.2) for $\mathfrak{B I}$ : Let $w_{X}, w_{Y}, w_{Z}, w_{\kappa}, w_{\lambda}, w_{\mu} \in \mathbb{N}$ be given. For each $n \in \mathbb{N}$ let $\mathfrak{B} \mathfrak{I}_{n}$ denote the $\mathbb{F}$-subspace of $\mathfrak{B I}$ spanned by $X^{i} Y^{j} Z^{k} \kappa^{r} \lambda^{s} \mu^{t}$ for all $i, j, k, r, s, t \in \mathbb{N}$ with

$$
w_{X} i+w_{Y} j+w_{Z} k+w_{\kappa} r+w_{\lambda} s+w_{\mu} t \leq n .
$$

We show that the sequence $\left\{\mathfrak{B I} \mathfrak{I}_{n}\right\}_{n \in \mathbb{N}}$ is an $\mathbb{N}$-filtration of $\mathfrak{B I}$ if and only if

$$
\max \left\{w_{Z}, w_{\kappa}\right\} \leq w_{X}+w_{Y}, \quad \max \left\{w_{X}, w_{\lambda}\right\} \leq w_{Y}+w_{Z}, \quad \max \left\{w_{Y}, w_{\mu}\right\} \leq w_{Z}+w_{X} .
$$

We apply the basis (1.1) for $\Re$ and the $\mathbb{N}$-filtration $\left\{\mathfrak{B I} \mathfrak{I}_{n}\right\}_{n \in \mathbb{N}}$ of $\mathfrak{B I}$ associated with

$$
\left(w_{X}, w_{Y}, w_{Z}, w_{\kappa}, w_{\lambda}, w_{\mu}\right)=(4,4,6,8,9,9)
$$

to conclude the injectivity of $\zeta$.

We regard the Racah algebra $\Re$ as an $\mathbb{F}$-subalgebra of $\mathfrak{B I}$ via $\zeta$. Let $\mathfrak{C}$ denote the commutative $\mathbb{F}$-subalgebra of $\Re$ generated by $\alpha, \beta, \gamma, \delta$. Extending the setting [15, Section 2], each element of

$$
D^{2}+A^{2}+B^{2}+\frac{(\delta+2)\{A, B\}-\left\{A^{2}, B\right\}-\left\{A, B^{2}\right\}}{2}+A(\beta-\delta)+B(\delta-\alpha)+\mathfrak{C}
$$

is called a Casimir element of $\Re$ [22]. Each Casimir element of $\Re$ is central in $\Re$. We locate the expressions for the $D_{6}$-symmetric Casimir elements [22, Section 5] of $\Re$ in terms of

$$
\iota=X+Y+Z
$$

and $\kappa, \lambda, \mu$. Note that $\iota, \kappa, \lambda, \mu$ are in the centralizer of $\Re$ in $\mathfrak{B} \mathfrak{I}$. Furthermore we apply the $\mathbb{N}$-filtration $\left\{\mathfrak{B} \mathfrak{I}_{n}\right\}_{n \in \mathbb{N}}$ of $\mathfrak{B I}$ associated with

$$
\left(w_{X}, w_{Y}, w_{Z}, w_{\kappa}, w_{\lambda}, w_{\mu}\right)=(1,1,2,0,0,0)
$$

to prove that for any Casimir element $\Omega$ of $\Re$ there exists a unique four-variable polynomial $P\left(x_{1}, x_{2}, x_{3}, x_{4}\right)$ over $\mathbb{F}$ such that

$$
\Omega=P(\iota, \kappa, \lambda, \mu) .
$$

The outline of this paper is as follows: In Sections 2 and 3 we present the required backgrounds on $\Re$ and $\mathfrak{B I}$, especially the basis (1.1) for $\Re$ and the criterion for $\left\{\mathfrak{B} \mathfrak{I}_{n}\right\}_{n \in \mathbb{N}}$ as an $\mathbb{N}$-filtration of $\mathfrak{B I}$. In Section 4 we review the homomorphism $\zeta: \Re \rightarrow \mathfrak{B I}$ and evaluate the image of $D$ under $\zeta$. In Section 5 we give the proof for the injectivity of $\zeta$. In Section 6 we show that each Casimir element of $\Re$ can be uniquely expressed as a polynomial in $\iota, \kappa, \lambda, \mu$ over $\mathbb{F}$. 


\section{$2 \quad$ The Racah algebra $\Re$}

Definition 2.1 ([7, 16, 19, 23]). The Racah algebra $\Re$ is an $\mathbb{F}$-algebra defined by generators and relations in the following way. The generators are $A, B, C, D$. The relations assert that

$$
[A, B]=[B, C]=[C, A]=2 D
$$

and each of

$$
[A, D]+A C-B A, \quad[B, D]+B A-C B, \quad[C, D]+C B-A C
$$

is central in $\Re$.

We define $\alpha, \beta, \gamma, \delta$ as the following elements of $\Re$ :

$$
\begin{aligned}
\alpha & =[A, D]+A C-B A, \\
\beta & =[B, D]+B A-C B, \\
\gamma & =[C, D]+C B-A C, \\
\delta & =A+B+C .
\end{aligned}
$$

Lemma 2.2 ([22, Lemma 3.2]). The following $(i)-($ iii $)$ hold:

(i) The $\mathbb{F}$-algebra $\Re$ is generated by $A, B, C$.

(ii) Each of $\alpha, \beta, \gamma, \delta$ is central in $\Re$.

(iii) The sum of $\alpha, \beta, \gamma$ is equal to zero.

Proposition 2.3. The $\mathbb{F}$-algebra $\Re$ has a presentation with generators $A, B, C, D, \alpha, \beta$ and relations

$$
\begin{aligned}
& B A=A B-2 D, \\
& C B=B C-2 D, \\
& C A=A C+2 D, \\
& D A=A D-A B+A C+2 D-\alpha, \\
& D B=B D-B C+A B-\beta, \\
& D C=C D-A C+B C-2 D+\alpha+\beta, \\
& \alpha A=A \alpha, \quad \alpha B=B \alpha, \quad \alpha C=C \alpha, \quad \alpha D=D \alpha, \quad \beta C=C \beta, \quad \beta D=D \beta, \quad \beta \alpha=\alpha \beta . \\
& \beta A=A \beta, \quad \beta B=B \beta, \quad \beta C
\end{aligned}
$$

Proof. Relations (2.6)-(2.8) are immediate from (2.1). Relation (2.9) follows from (2.2), (2.6). Relation (2.10) follows from (2.3), (2.6) and (2.7). Relation (2.11) follows from (2.4), (2.7) and Lemma 2.2(iii). Relations (2.12) and (2.13) follow from Lemma 2.2(ii).

Theorem 2.4. The elements

$$
A^{i} B^{j} C^{k} D^{\ell} \alpha^{r} \beta^{s} \quad \text { for all } i, j, k, \ell, r, s \in \mathbb{N}
$$

are an $\mathbb{F}$-basis $\Re$.

Proof. To prove the result we invoke the diamond lemma [1, Theorem 1.2]. The relations (2.6)-(2.13) are regarded as a reduction system. The $\mathbb{F}$-linear combinations of (2.14) are exactly the irreducible elements under the reduction system. There are no inclusion ambiguities in the 
reduction system. The nontrivial overlap ambiguities involve the words $C B A, D B A, D C B$, $D C A$. In any reduction ways, we eventually obtain that

$$
\begin{aligned}
C B A= & A B C+2 A B-2 B C-2 A D+2 B D-2 C D-2 \beta, \\
D B A= & A B D-2 D^{2}+A^{2} B-A B^{2}-2 A D+2 B D+2 A B-2 B C-A \beta-B \alpha-2 \beta, \\
D C B= & B C D-2 D^{2}+B^{2} C-B C^{2}-2 B D+2 C D-2 A C+2 B C+B \alpha+B \beta-C \beta \\
& -4 D+2 \alpha+2 \beta, \\
D C A= & A C D+2 D^{2}-A^{2} C+A C^{2}-2 A D-2 A C+2 B C+2 C D+A \alpha+A \beta-C \alpha \\
& -4 D+2 \alpha+2 \beta .
\end{aligned}
$$

Hence each of the overlap ambiguities is resolvable.

Let $M$ denote the free monoid with the alphabet set $S=\{A, B, C, D, \alpha, \beta\}$. Let $\ell: M \rightarrow \mathbb{N}$ denote the length function of $M$. Consider an element $w=s_{1} s_{2} \cdots s_{n} \in M$ where $s_{1}, s_{2}, \ldots, s_{n} \in S$. An operation on $w$ is called an elementary operation if it is one of the following actions on $w$ :

- We interchange $s_{i}$ and $s_{j}$ where $1 \leq i<j \leq n$ and the position of $s_{j}$ is left to the position of $s_{i}$ in the list

$A, \quad B, \quad C, \quad D, \quad \alpha, \quad \beta$.

- Choose $s_{i} \in\{B, C, D\}$ and replace $s_{i}$ by the left neighbor of $s_{i}$ in the list

$$
A, \quad B, \quad C, \quad D \text {. }
$$

We define a binary relation $\preceq$ on $M$ as follows: For any $u, w \in M$ we say that $u \rightarrow w$ whenever $\ell(u)<\ell(w)$ or $u$ is obtained from $w$ by an elementary operation. For any $u, w \in M$ we define $u \preceq w$ if there exist $u_{0}, u_{1}, \ldots, u_{k} \in M$ with $k \in \mathbb{N}$ such that

$$
u=u_{0} \rightarrow u_{1} \rightarrow \cdots \rightarrow u_{k-1} \rightarrow u_{k}=w .
$$

By construction $\preceq$ is a partial order relation on $M$ satisfying the descending chain condition. Moreover $\preceq$ is a monoid partial order on $M$ compatible with the reduction system (2.6)-(2.13). Therefore, by diamond lemma the monomials (2.14) form an $\mathbb{F}$-basis for $\Re$.

Recall that the dihedral group $D_{6}$ has a presentation with generators $\sigma, \tau$ and relations

$$
\sigma^{2}=1, \quad \tau^{6}=1, \quad(\sigma \tau)^{2}=1 .
$$

Proposition 2.5 ([22, Propositions 4.1 and 4.3]). There exists a unique $D_{6}$-action on $\Re$ such that ( $i)$, (ii) hold:

(i) $\sigma$ acts on $\Re$ as an $\mathbb{F}$-algebra antiautomorphism of $\Re$ given in the following way:

\begin{tabular}{c|cccc|cccc}
$u$ & $A$ & $B$ & $C$ & $D$ & $\alpha$ & $\beta$ & $\gamma$ & $\delta$ \\
\hline$\sigma(u)$ & $B$ & $A$ & $C$ & $D$ & $-\beta$ & $-\alpha$ & $-\gamma$ & $\delta$
\end{tabular}

(ii) $\tau$ acts on $\Re$ as an $\mathbb{F}$-algebra antiautomorphism of $\Re$ given in the following way:

\begin{tabular}{c|cccc|cccc}
$u$ & $A$ & $B$ & $C$ & $D$ & $\alpha$ & $\beta$ & $\gamma$ & $\delta$ \\
\hline$\tau(u)$ & $B$ & $C$ & $A$ & $-D$ & $\beta$ & $\gamma$ & $\alpha$ & $\delta$
\end{tabular}

Moreover the $D_{6}$-action on $\Re$ is faithful.

Let $\mathfrak{C}$ denote the $\mathbb{F}$-subalgebra of $\Re$ generated by $\alpha, \beta, \gamma, \delta$. It follows from Lemma 2.2(ii) that $\mathfrak{C}$ is commutative. 
Definition 2.6 ([22, Definition 5.2]). The coset

$$
D^{2}+A^{2}+B^{2}+\frac{(\delta+2)\{A, B\}-\left\{A^{2}, B\right\}-\left\{A, B^{2}\right\}}{2}+A(\beta-\delta)+B(\delta-\alpha)+\mathfrak{C}
$$

is called the Casimir class of $\Re$. Each element of the Casimir class of $\Re$ is called a Casimir element of $\Re$.

Define

$$
\begin{aligned}
& \Omega_{A}=D^{2}+\frac{B A C+C A B}{2}+A^{2}+B \gamma-C \beta-A \delta, \\
& \Omega_{B}=D^{2}+\frac{C B A+A B C}{2}+B^{2}+C \alpha-A \gamma-B \delta, \\
& \Omega_{C}=D^{2}+\frac{A C B+B C A}{2}+C^{2}+A \beta-B \alpha-C \delta .
\end{aligned}
$$

Note that $\Omega_{A}, \Omega_{B}, \Omega_{C}$ are mutually distinct [22, Corollary 6.5].

Lemma 2.7 ([22, Proposition 3.7]). Each of $\Omega_{A}, \Omega_{B}, \Omega_{C}$ is a Casimir element.

Lemma 2.8 ([22, Lemma 3.6]). The set $\left\{\Omega_{A}, \Omega_{B}, \Omega_{C}\right\}$ is invariant under the $D_{6}$-action on $\Re$. Moreover the restrictions of $\sigma$ and $\tau$ to $\left\{\Omega_{A}, \Omega_{B}, \Omega_{C}\right\}$ are as follows:

\begin{tabular}{c|ccc}
$u$ & $\Omega_{A}$ & $\Omega_{B}$ & $\Omega_{C}$ \\
\hline$\sigma(u)$ & $\Omega_{B}$ & $\Omega_{A}$ & $\Omega_{C}$ \\
$\tau(u)$ & $\Omega_{B}$ & $\Omega_{C}$ & $\Omega_{A}$
\end{tabular}

Definition 2.9 ([22, Section 5]). The elements $\Omega_{A}, \Omega_{B}, \Omega_{C}$ are called the $D_{6}$-symmetric Casimir elements of $\Re$.

\section{The Bannai-Ito algebra $\mathfrak{B} \mathfrak{I}$}

Definition 3.1. The Bannai-Ito algebra $\mathfrak{B} \mathfrak{I}$ is an $\mathbb{F}$-algebra defined by generators and relations. The generators are $X, Y, Z$ and the relations assert that each of $\{X, Y\}-Z,\{Y, Z\}-X$, $\{Z, X\}-Y$ is central in $\mathfrak{B} \mathfrak{I}$.

We define $\iota, \kappa, \lambda, \mu$ as the following elements of $\mathfrak{B I}$ :

$$
\begin{aligned}
& \iota=X+Y+Z, \\
& \kappa=\{X, Y\}-Z, \\
& \lambda=\{Y, Z\}-X, \\
& \mu=\{Z, X\}-Y .
\end{aligned}
$$

Proposition 3.2. There exists a unique $D_{6}$-action on $\mathfrak{B I}$ such that $(i)$, (ii) hold:

(i) $\sigma$ acts on $\mathfrak{B I}$ as an $\mathbb{F}$-algebra antiautomorphism of $\mathfrak{B I}$ given in the following way:

\begin{tabular}{c|ccc|cccc}
$u$ & $X$ & $Y$ & $Z$ & $\iota$ & $\kappa$ & $\lambda$ & $\mu$ \\
\hline$\sigma(u)$ & $Y$ & $X$ & $Z$ & $\iota$ & $\kappa$ & $\mu$ & $\lambda$
\end{tabular}

(ii) $\tau$ acts on $\mathfrak{B I}$ as an $\mathbb{F}$-algebra antiautomorphism of $\mathfrak{B I}$ given in the following way: 


\begin{tabular}{c|ccc|cccc}
$u$ & $X$ & $Y$ & $Z$ & $\iota$ & $\kappa$ & $\lambda$ & $\mu$ \\
\hline$\tau(u)$ & $Y$ & $Z$ & $X$ & $\iota$ & $\lambda$ & $\mu$ & $\kappa$
\end{tabular}

Moreover the $D_{6}$-action on $\mathfrak{B I}$ is faithful.

Proof. It is straightforward to verify the existence of the $D_{6}$-action on $\mathfrak{B I}$ by using (2.15) and Definition 3.1. Since $D_{6}$ is generated by $\sigma$ and $\tau$ the uniqueness follows. The $\mathbb{F}$-algebra antiautomorphism of $\mathfrak{B I}$ given in (ii) is of order 6 . It follows from [22, Lemma 4.2] that the $D_{6}$-action on $\mathfrak{B I}$ is faithful.

Proposition 3.3. The $\mathbb{F}$-algebra $\mathfrak{B} \mathfrak{I}$ has a presentation with generators $X, Y, Z, \kappa, \lambda$, $\mu$ and relations

$$
\begin{aligned}
& Y X=-X Y+Z+\kappa, \quad Z Y=-Y Z+X+\lambda, \quad Z X=-X Z+Y+\mu, \\
& \kappa X=X \kappa, \quad \kappa Y=Y \kappa, \quad \kappa Z=Z \kappa, \\
& \lambda X=X \lambda, \quad \lambda Y=Y \lambda, \quad \lambda Z=Z \lambda, \quad \lambda \kappa=\kappa \lambda, \quad \\
& \mu X=X \mu, \quad \mu Y=Y \mu, \quad \mu Z=Z \mu, \quad \mu \kappa=\kappa \mu, \quad \mu \lambda=\lambda \mu .
\end{aligned}
$$

Proof. Immediate from Definition 3.1.

Applying the diamond lemma to Proposition 3.3, we obtain the following Poincaré-BirkhoffWitt basis for $\mathfrak{B I}$. Since the argument is similar to the proof of Theorem 2.4, we omit the proof here.

Theorem 3.4. The elements

$$
X^{i} Y^{j} Z^{k} \kappa^{r} \lambda^{s} \mu^{t} \quad \text { for all } i, j, k, r, s, t \in \mathbb{N}
$$

form an $\mathbb{F}$-basis for $\mathfrak{B} \mathfrak{I}$.

Let $\mathcal{A}$ denote an $\mathbb{F}$-algebra and let $\mathcal{H}, \mathcal{K}$ denote two $\mathbb{F}$-subspaces of $\mathcal{A}$. The product $\mathcal{H} \cdot \mathcal{K}$ is meant to be the $\mathbb{F}$-subspace of $\mathcal{A}$ spanned by $h \cdot k$ for all $h \in \mathcal{H}$ and all $k \in \mathcal{K}$. Recall that an $\mathbb{N}$-filtration of $\mathcal{A}$ is a sequence $\left\{\mathcal{A}_{n}\right\}_{n \in \mathbb{N}}$ of $\mathbb{F}$-subspaces of $\mathcal{A}$ satisfies the following conditions:

(N1) $\bigcup_{n \in \mathbb{N}} \mathcal{A}_{n}=\mathcal{A}$.

(N2) $\mathcal{A}_{n} \subseteq \mathcal{A}_{n+1}$ for all $n \in \mathbb{N}$.

(N3) $\mathcal{A}_{m} \cdot \mathcal{A}_{n} \subseteq \mathcal{A}_{m+n}$ for all $m, n \in \mathbb{N}$.

For convenience we always let $\mathcal{A}_{-1}$ denote the zero subspace of $\mathcal{A}$.

We consider the following $\mathbb{F}$-subspaces of $\mathfrak{B} \mathfrak{I}$ induced from Theorem 3.4: Let $w_{X}, w_{Y}, w_{Z}$, $w_{\kappa}, w_{\lambda}, w_{\mu}$ denote the nonnegative integers. For each $n \in \mathbb{N}$ let $\mathfrak{B I}_{n}$ denote the $\mathbb{F}$-subspace of $\mathfrak{B} \mathfrak{I}$ spanned by $X^{i} Y^{j} Z^{k} \kappa^{r} \lambda^{s} \mu^{t}$ for all $i, j, k, r, s, t \in \mathbb{N}$ with

$$
w_{X} i+w_{Y} j+w_{Z} k+w_{\kappa} r+w_{\lambda} s+w_{\mu} t \leq n .
$$

We call $\left\{\mathfrak{B I}_{n}\right\}_{n \in \mathbb{N}}$ the $\mathbb{F}$-subspaces of $\mathfrak{B I}$ associated with $\left(w_{X}, w_{Y}, w_{Z}, w_{\kappa}, w_{\lambda}, w_{\mu}\right)$. In what follows we give a simple criterion for the above $\mathbb{F}$-subspaces of $\mathfrak{B} \mathfrak{I}$ to be an $\mathbb{N}$-filtration of $\mathfrak{B} \mathfrak{I}$.

Theorem 3.5. Let $w_{X}, w_{Y}, w_{Z}, w_{\kappa}, w_{\lambda}, w_{\mu} \in \mathbb{N}$. Let $\left\{\mathfrak{B} \mathfrak{I}_{n}\right\}_{n \in \mathbb{N}}$ denote the $\mathbb{F}$-subspaces of $\mathfrak{B I}$ associated with $\left(w_{X}, w_{Y}, w_{Z}, w_{\kappa}, w_{\lambda}, w_{\mu}\right)$. Then $\left\{\mathfrak{B I}_{n}\right\}_{n \in \mathbb{N}}$ is an $\mathbb{N}$-filtration of $\mathfrak{B I}$ if and only if

$$
\begin{aligned}
& \max \left\{w_{Z}, w_{\kappa}\right\} \leq w_{X}+w_{Y}, \\
& \max \left\{w_{X}, w_{\lambda}\right\} \leq w_{Y}+w_{Z}, \\
& \max \left\{w_{Y}, w_{\mu}\right\} \leq w_{Z}+w_{X} .
\end{aligned}
$$


Proof. $(\Rightarrow)$ By the construction of $\left\{\mathfrak{B I} \mathfrak{I}_{n}\right\}_{n \in \mathbb{N}}$ and Theorem 3.4 the element

$$
Z+\kappa \notin \mathfrak{B} \mathfrak{I}_{\max \left\{w_{Z}, w_{\kappa}\right\}-1} \cdot
$$

On the other hand, by (N3) we have $\{X, Y\} \in \mathfrak{B I}_{w_{X}+w_{Y}}$. The equation (3.2) implies

$$
Z+\kappa=\{X, Y\} .
$$

By the above comments we see that $\mathfrak{B} \mathfrak{I}_{w_{X}+w_{Y}}$ contains $Z+\kappa$ which is not in $\mathfrak{B} \mathfrak{I}_{\max \left\{w_{Z}, w_{\kappa}\right\}-1}$. Combined with (N2) the inequality (3.6) follows. The inequalities (3.7) and (3.8) follow by similar arguments.

$(\Leftarrow)$ Condition $(\mathrm{N} 1)$ is immediate from Theorem 3.4. Condition (N2) is immediate from the construction of $\left\{\mathfrak{B I}_{n}\right\}_{n \in \mathbb{N}}$. Set $S=\{X, Y, Z, \kappa, \lambda, \mu\}$. For all $n \in \mathbb{N}$, let $I_{n}$ denote the set of all $(i, j, k, r, s, t) \in \mathbb{N}^{6}$ with $w_{X} i+w_{Y} j+w_{Z} k+w_{\kappa} r+w_{\lambda} s+w_{\mu} t \leq n$. Let $M$ denote the free monoid with the alphabet set $S$. There exists a unique monoid homomorphism $\tilde{w}: M \rightarrow \mathbb{N}$ such that

$$
\tilde{w}(u)=w_{u} \quad \text { for all } u \in S .
$$

By (3.6)-(3.8), for each relation of Proposition 3.3, the value of $\tilde{w}$ on the monomial in the lefthand side is greater than or equal to those in the right-hand side. Thus, for all $m, n \in \mathbb{N}$ and for all $\left(i^{\prime}, j^{\prime}, k^{\prime}, r^{\prime}, s^{\prime}, t^{\prime}\right) \in I_{m}$ and $\left(i^{\prime \prime}, j^{\prime \prime}, k^{\prime \prime}, r^{\prime \prime}, s^{\prime \prime}, t^{\prime \prime}\right) \in I_{n}$ the product

$$
X^{i^{\prime}} Y^{j^{\prime}} Z^{k^{\prime}} \kappa^{r^{\prime}} \lambda^{s^{\prime}} \mu^{t^{\prime}} \cdot X^{i^{\prime \prime}} Y^{j^{\prime \prime}} Z^{k^{\prime \prime}} \kappa^{r^{\prime \prime}} \lambda^{s^{\prime \prime}} \mu^{t^{\prime \prime}}
$$

is equal to an $\mathbb{F}$-linear combination of $X^{i} Y^{j} Z^{k} \kappa^{r} \lambda^{s} \mu^{t}$ for all $(i, j, k, r, s, t) \in I_{m+n}$. In other words (N3) holds. The theorem follows.

\section{The homomorphism $\zeta: \Re \rightarrow \mathfrak{B} \mathfrak{I}$}

According to $[16$, Section 2] there exists an $\mathbb{F}$-algebra homomorphism $\zeta: \Re \rightarrow \mathfrak{B I}$ and the images of $A, B, C, \alpha, \beta, \gamma, \delta$ under $\zeta$ are as follows:

Theorem 4.1 ([16]). There exists a unique $\mathbb{F}$-algebra homomorphism $\zeta: \Re \rightarrow \mathfrak{B I}$ that sends

$$
\begin{aligned}
& A \mapsto \frac{(2 X-3)(2 X+1)}{16}, \quad B \mapsto \frac{(2 Y-3)(2 Y+1)}{16}, \quad C \mapsto \frac{(2 Z-3)(2 Z+1)}{16}, \\
& \alpha \mapsto \frac{(2 \iota-\kappa-\mu-3)(\kappa-\mu)}{64}, \quad \beta \mapsto \frac{(2 \iota-\lambda-\kappa-3)(\lambda-\kappa)}{64}, \\
& \gamma \mapsto \frac{(2 \iota-\mu-\lambda-3)(\mu-\lambda)}{64}, \quad \delta \mapsto \frac{\iota^{2}-2 \iota-\kappa-\lambda-\mu}{4}-\frac{9}{16} .
\end{aligned}
$$

We are now going to evaluate the image of $D$ under $\zeta$.

\section{Lemma 4.2.}

(i) The following equations hold in $\mathfrak{B} \mathfrak{I}$ :

$$
\begin{aligned}
& {\left[X^{2}, Y\right]=[X, Z], \quad\left[Y^{2}, Z\right]=[Y, X], \quad\left[Z^{2}, X\right]=[Z, Y],} \\
& {\left[Y^{2}, X\right]=[Y, Z], \quad\left[Z^{2}, Y\right]=[Z, X], \quad\left[X^{2}, Z\right]=[X, Y] .}
\end{aligned}
$$

(ii) The following elements of $\mathfrak{B} \mathfrak{I}$ are equal:

$$
\begin{aligned}
& \{X,[Z, Y]\}, \quad\{Y,[X, Z]\}, \quad\{Z,[Y, X]\}, \\
& {\left[X^{2}, Y^{2}\right], \quad\left[Y^{2}, Z^{2}\right], \quad\left[Z^{2}, X^{2}\right] .}
\end{aligned}
$$


Proof. (i) Since $\kappa$ is central in $\mathfrak{B I}$ and by (3.2) it follows that

$$
[X, Z]=[X,\{X, Y\}]
$$

Observe that $[X,\{X, Y\}]=\left[X^{2}, Y\right]$. Therefore

$$
\left[X^{2}, Y\right]=[X, Z]
$$

Applying Proposition 3.2 to (4.1) yields the remaining equations in (i).

(ii) By Proposition 3.2(ii) it suffices to show that

$$
\begin{aligned}
& \{X,[Z, Y]\}=\{Y,[X, Z]\}, \\
& \{X,[Z, Y]\}=\left[X^{2}, Y^{2}\right] .
\end{aligned}
$$

With trivial cancellations we obtain

$$
\{X,[Z, Y]\}-\{Y,[X, Z]\}=[Z,\{Y, X\}]
$$

Since $\kappa$ is central in $\mathfrak{B I}$ and by (3.2) the element $Z$ commutes with $\{Y, X\}$. Hence the right-hand side of (4.4) is zero. Therefore (4.2) follows. Using (3.2) twice we find that

$$
X^{2} Y^{2}=X Y^{2} X+X Z Y-X Y Z \text {. }
$$

By Proposition 3.2(ii), $\tau^{3}$ is an $\mathbb{F}$-algebra antiautomorphism of $\mathfrak{B} \mathfrak{I}$ that fixes $X, Y, Z$. Thus, applying $\tau^{3}$ to $(4.5)$ yields that

$$
Y^{2} X^{2}=X Y^{2} X+Y Z X-Z Y X .
$$

Subtracting (4.6) from (4.5) yields (4.3). Hence (ii) follows.

For convenience we let $L$ denote the common element of $\mathfrak{B} \mathfrak{I}$ from Lemma 4.2(ii).

Proposition 4.3. The image of $D$ under $\zeta$ is equal to

$$
\frac{[X, Y]+[Y, Z]+[Z, X]+L}{32} .
$$

Proof. By (2.1) we have $2 D^{\zeta}=\left[A^{\zeta}, B^{\zeta}\right]$. A direct calculation yields that $\left[A^{\zeta}, B^{\zeta}\right]$ is equal to

$$
\frac{\left[X^{2}, Y^{2}\right]+[X, Y]+\left[Y^{2}, X\right]+\left[Y, X^{2}\right]}{16} .
$$

By Lemma 4.2(i), $\left[Y^{2}, X\right]=[Y, Z]$ and $\left[Y, X^{2}\right]=[Z, X]$. By Lemma 4.2(ii), $\left[X^{2}, Y^{2}\right]=L$. The proposition follows.

Corollary 4.4. For each $g \in D_{6}$ the following diagram commutes:

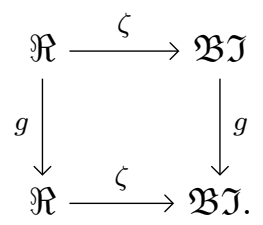

Proof. It is routine to verify the corollary by using Propositions 2.5, 3.2 and Theorem 4.1. 
We end this section with a comment: Recall from $[18,21]$ that a universal analogue of the additive DAHA (double affine Hecke algebra) of type $\left(C_{1}^{\vee}, C_{1}\right)$, denoted by $\mathfrak{H}$ here, is an $\mathbb{F}$ algebra generated by $t_{0}, t_{1}, t_{0}^{\vee}, t_{1}^{\vee}$ and the relations assert that

$$
t_{0}+t_{1}+t_{0}^{\vee}+t_{1}^{\vee}=-1
$$

and each of $t_{0}^{2}, t_{1}^{2}, t_{0}^{\vee 2}, t_{1}^{\vee 2}$ is central in $\mathfrak{H}$. By [18, Proposition 2] there exists an $\mathbb{F}$-algebra isomorphism $\downarrow$ : $\mathfrak{B I} \rightarrow \mathfrak{H}$ that sends

$$
\begin{aligned}
& X \mapsto t_{0}+t_{1}+\frac{1}{2}, \quad Y \mapsto t_{0}+t_{0}^{\vee}+\frac{1}{2}, \quad Z \mapsto t_{0}+t_{1}^{\vee}+\frac{1}{2}, \quad \iota \mapsto 2 t_{0}+\frac{1}{2}, \\
& \kappa \mapsto t_{0}^{2}-t_{1}^{2}-t_{0}^{\vee 2}+t_{1}^{\vee 2}, \quad \lambda \mapsto t_{0}^{2}-t_{0}^{\vee 2}-t_{1}^{\vee 2}+t_{1}^{2}, \quad \mu \mapsto t_{0}^{2}-t_{1}^{\vee 2}-t_{1}^{2}+t_{0}^{\vee 2} .
\end{aligned}
$$

The universal Askey-Wilson algebra [24] and the universal DAHA of type $\left(C_{1}^{\vee}, C_{1}\right)$ [25] are the $q$-analogues of $\Re$ and $\mathfrak{H}$, respectively. Therefore [25, Theorem 4.1] is a $q$-analogue of the homomorphism $\downarrow \circ \zeta: \Re \rightarrow \mathfrak{H}$. Note that $\natural \circ \zeta$ sends

$$
\begin{array}{ll}
A \mapsto \frac{\left(t_{1}^{\vee}+t_{0}^{\vee}\right)\left(t_{1}^{\vee}+t_{0}^{\vee}+2\right)}{4}, \quad B \mapsto \frac{\left(t_{1}+t_{1}^{\vee}\right)\left(t_{1}+t_{1}^{\vee}+2\right)}{4}, \\
C \mapsto \frac{\left(t_{0}^{\vee}+t_{1}\right)\left(t_{0}^{\vee}+t_{1}+2\right)}{4}, \\
\alpha \mapsto \frac{\left(t_{1}^{\vee 2}-t_{0}^{\vee 2}\right)\left(t_{1}^{2}-t_{0}^{2}+2 t_{0}-1\right)}{16}, \quad \beta \mapsto \frac{\left(t_{1}^{2}-t_{1}^{\vee 2}\right)\left(t_{0}^{\vee 2}-t_{0}^{2}+2 t_{0}-1\right)}{16}, \\
\gamma \mapsto \frac{\left(t_{0}^{\vee 2}-t_{1}^{2}\right)\left(t_{1}^{\vee 2}-t_{0}^{2}+2 t_{0}-1\right)}{16}, \quad \delta \mapsto \frac{t_{0}^{2}+t_{1}^{2}+t_{0}^{\vee 2}+t_{1}^{\vee 2}}{4}-\frac{t_{0}}{2}-\frac{3}{4} .
\end{array}
$$

\section{The injectivity of $\zeta$}

Throughout this section, we let $\left\{\mathfrak{B} \mathfrak{I}_{n}\right\}_{n \in \mathbb{N}}$ denote the $\mathbb{F}$-subspaces of $\mathfrak{B I}$ associated with

$$
\left(w_{X}, w_{Y}, w_{Z}, w_{\kappa}, w_{\lambda}, w_{\mu}\right)=(4,4,6,8,9,9) .
$$

Since the number sequence (5.1) satisfies (3.6)-(3.8), it follows from Theorem 3.5 that $\left\{\mathfrak{B} \mathfrak{I}_{n}\right\}_{n \in \mathbb{N}}$ is an $\mathbb{N}$-filtration of $\mathfrak{B I}$.

\section{Lemma 5.1.}

(i) For any even integer $n \geq 0$ the following equations hold:

$$
\begin{array}{ll}
Y^{n} X=X Y^{n} & \left(\bmod \mathfrak{B I}_{4 n+3}\right), \\
X^{n} Y=Y X^{n} & \left(\bmod \mathfrak{B I}_{4 n+3}\right), \\
Z^{n} Y=Y Z^{n} & \left(\bmod \mathfrak{B} \mathfrak{I}_{6 n+3}\right), \\
Y^{n} Z=Z Y^{n} & \left(\bmod \mathfrak{B I}_{4 n+5}\right), \\
X^{n} Z=Z X^{n} \quad\left(\bmod \mathfrak{B I}_{4 n+5}\right), \\
Z^{n} X=X Z^{n} \quad\left(\bmod \mathfrak{B I}_{6 n+3}\right) .
\end{array}
$$

(ii) For any odd integer $n \geq 1$ the following equations hold:

$$
\begin{aligned}
Y^{n} X & =-X Y^{n}+\kappa Y^{n-1} \quad\left(\bmod \mathfrak{B} \mathfrak{I}_{4 n+3}\right), \\
X^{n} Y & =-Y X^{n}+\kappa X^{n-1} \quad\left(\bmod \mathfrak{B} \mathfrak{I}_{4 n+3}\right), \\
Y^{n} Z & =-Z Y^{n} \quad\left(\bmod \mathfrak{B} \mathfrak{I}_{4 n+5}\right), \\
Z^{n} Y & =-Y Z^{n} \quad\left(\bmod \mathfrak{B} \mathfrak{I}_{6 n+3}\right), \\
X^{n} Z & =-Z X^{n} \quad\left(\bmod \mathfrak{B I}_{4 n+5}\right), \\
Z^{n} X & =-X Z^{n} \quad\left(\bmod \mathfrak{B I}_{6 n+3}\right) .
\end{aligned}
$$


Proof. All equations are established by routine inductions and using (3.2)-(3.4).

\section{Lemma 5.2.}

(i) For any integer $n \geq 0$ the following equations hold:

$$
\begin{aligned}
\left(A^{\zeta}\right)^{n} & =\left(\frac{X}{2}\right)^{2 n} \quad\left(\bmod \mathfrak{B} \mathfrak{I}_{8 n-1}\right), \\
\left(B^{\zeta}\right)^{n} & =\left(\frac{Y}{2}\right)^{2 n} \quad\left(\bmod \mathfrak{B} \mathfrak{I}_{8 n-1}\right), \\
\left(C^{\zeta}\right)^{n} & =\left(\frac{Z}{2}\right)^{2 n} \quad\left(\bmod \mathfrak{B} \mathfrak{I}_{12 n-1}\right), \\
\left(\alpha^{\zeta}\right)^{n} & =\left(\frac{\mu}{8}\right)^{2 n} \quad\left(\bmod \mathfrak{B} \mathfrak{I}_{18 n-1}\right), \\
\left(\beta^{\zeta}\right)^{n} & =(-1)^{n}\left(\frac{\lambda}{8}\right)^{2 n} \quad\left(\bmod \mathfrak{B} \mathfrak{I}_{18 n-1}\right) .
\end{aligned}
$$

(ii) For any even integer $n \geq 0$ the following equation holds:

$$
\left(D^{\zeta}\right)^{n}=\frac{1}{16^{n}} \sum_{i=0}^{\frac{n}{2}}(-4)^{i}\left(\begin{array}{c}
\frac{n}{2} \\
i
\end{array}\right) X^{2 i} Y^{2 i} \kappa^{n-2 i} Z^{n} \quad\left(\bmod \mathfrak{B} \mathfrak{I}_{14 n-1}\right) .
$$

(iii) For any odd integer $n \geq 1$ the following equation holds:

$$
\begin{aligned}
& \left(D^{\zeta}\right)^{n}=\frac{1}{16^{n}} \sum_{i=0}^{\frac{n-1}{2}}(-4)^{i}\left(\begin{array}{c}
\frac{n-1}{2} \\
i
\end{array}\right)\left(X^{2 i} Y^{2 i} \kappa^{n-2 i}-2 X^{2 i+1} Y^{2 i+1} \kappa^{n-2 i-1}\right) Z^{n} \\
& \left(\bmod \mathfrak{B} \mathfrak{I}_{14 n-1}\right) .
\end{aligned}
$$

Proof. (i) Immediate from Theorem 4.1 and the construction of $\left\{\mathfrak{B I}_{n}\right\}_{n \in \mathbb{N}}$.

(ii) It follows from Proposition 4.3 that

$$
D^{\zeta}=\frac{L}{32} \quad\left(\bmod \mathfrak{B} \mathfrak{I}_{13}\right) .
$$

Evaluating $L \bmod \mathfrak{B} \mathfrak{I}_{13}$ by using Lemma 4.2(ii) and Lemma 5.1(ii) yields that

$$
D^{\zeta}=\frac{Z \kappa}{16}-\frac{X Y Z}{8} \quad\left(\bmod \mathfrak{B I}_{13}\right) .
$$

Squaring the equation (5.2) a direct calculation shows that

$$
\left(D^{\zeta}\right)^{2}=\frac{Z^{2} \kappa^{2}}{256}-\frac{X^{2} Y^{2} Z^{2}}{64}\left(\bmod \mathfrak{B I}_{27}\right) .
$$

It follows from Lemma 5.1(i) that

$$
Z^{2} \cdot X^{2} Y^{2} Z^{2}=X^{2} Y^{2} Z^{2} \cdot Z^{2} \quad\left(\bmod \mathfrak{B I}_{39}\right) .
$$

Now it is routine to derive (ii) by using (5.3) and (5.4).

(iii) To get (iii), one may multiply (5.2) by the equation from (ii) and simplify the resulting equation by using Lemma 5.1(i). 
Lemma 5.3. Let $i, j, k, \ell, n, r, s \in \mathbb{N}$ with $8 i+8 j+12 k+14 \ell+18 r+18 s=n$. Then the following (i)-(iii) hold:

(i) For all $i^{\prime}, j^{\prime}, k^{\prime}, r^{\prime}, s^{\prime}, t^{\prime} \in \mathbb{N}$ with $4 i^{\prime}+4 j^{\prime}+6 k^{\prime}+8 r^{\prime}+9 s^{\prime}+9 t^{\prime}=n$ and $r^{\prime}>\ell$, the coefficient of

$$
X^{i^{\prime}} Y^{j^{\prime}} Z^{k^{\prime}} \kappa^{r^{\prime}} \lambda^{s^{\prime}} \mu^{t^{\prime}}
$$

in $\left(A^{\zeta}\right)^{i}\left(B^{\zeta}\right)^{j}\left(C^{\zeta}\right)^{k}\left(D^{\zeta}\right)^{\ell}\left(\alpha^{\zeta}\right)^{r}\left(\beta^{\zeta}\right)^{s}$ with respect to the $\mathbb{F}$-basis (3.5) for $\mathfrak{B} \mathfrak{I}$ is zero.

(ii) For all $i^{\prime}, j^{\prime}, k^{\prime}, r^{\prime}, s^{\prime}, t^{\prime} \in \mathbb{N}$ with $4 i^{\prime}+4 j^{\prime}+6 k^{\prime}+8 r^{\prime}+9 s^{\prime}+9 t^{\prime}=n$ and $r^{\prime}=\ell$, the coefficient of

$$
X^{i^{\prime}} Y^{j^{\prime}} Z^{k^{\prime}} \kappa^{r^{\prime}} \lambda^{s^{\prime}} \mu^{t^{\prime}}
$$

in $\left(A^{\zeta}\right)^{i}\left(B^{\zeta}\right)^{j}\left(C^{\zeta}\right)^{k}\left(D^{\zeta}\right)^{\ell}\left(\alpha^{\zeta}\right)^{r}\left(\beta^{\zeta}\right)^{s}$ with respect to the $\mathbb{F}$-basis (3.5) for $\mathfrak{B I}$ is nonzero if and only if

$$
\left(i^{\prime}, j^{\prime}, k^{\prime}, s^{\prime}, t^{\prime}\right)=(2 i, 2 j, 2 k+\ell, 2 s, 2 r)
$$

(iii) The coefficient of

$$
X^{2 i} Y^{2 j} Z^{2 k+\ell} \kappa^{\ell} \lambda^{2 s} \mu^{2 r}
$$

in $\left(A^{\zeta}\right)^{i}\left(B^{\zeta}\right)^{j}\left(C^{\zeta}\right)^{k}\left(D^{\zeta}\right)^{\ell}\left(\alpha^{\zeta}\right)^{r}\left(\beta^{\zeta}\right)^{s}$ with respect to the $\mathbb{F}$-basis (3.5) for $\mathfrak{B I}$ is

$$
(-1)^{s} 4^{-i-j-k-2 \ell-3 r-3 s} \text {. }
$$

Proof. Using Lemmas 5.1(i) and 5.2 one may express

$$
\left(A^{\zeta}\right)^{i}\left(B^{\zeta}\right)^{j}\left(C^{\zeta}\right)^{k}\left(D^{\zeta}\right)^{\ell}\left(\alpha^{\zeta}\right)^{r}\left(\beta^{\zeta}\right)^{s}+\mathfrak{B I}_{n-1}
$$

as an $\mathbb{F}$-linear combination of $X^{i^{\prime}} Y^{j^{\prime}} Z^{k^{\prime}} \kappa^{r^{\prime}} \lambda^{s^{\prime}} \mu^{t^{\prime}}+\mathfrak{B} \mathfrak{I}_{n-1}$ for all $i^{\prime}, j^{\prime}, k^{\prime}, r^{\prime}, s^{\prime}, t^{\prime} \in \mathbb{N}$ with $4 i^{\prime}+$ $4 j^{\prime}+6 k^{\prime}+8 r^{\prime}+9 s^{\prime}+9 t^{\prime}=n$. The lemma follows from the expression.

Theorem 5.4. The homomorphism $\zeta: \Re \rightarrow \mathfrak{B I}$ is injective.

Proof. Suppose on the contrary that there exists a nonzero element $I$ in the kernel of $\zeta$. For all $i, j, k, \ell, r, s \in \mathbb{N}$ let $c(i, j, k, \ell, r, s)$ denote the coefficient of

$$
A^{i} B^{j} C^{k} D^{\ell} \alpha^{r} \beta^{s}
$$

in $I$ with respect to the $\mathbb{F}$-basis (2.14) for $\Re$. Let $S$ denote the set of all $(i, j, k, \ell, r, s) \in \mathbb{N}^{6}$ with $c(i, j, k, \ell, r, s) \neq 0$. For each $n \in \mathbb{N}$ we let $S(n)$ denote the set of all $(i, j, k, \ell, r, s) \in S$ with $8 i+8 j+12 k+14 \ell+18 r+18 s=n$. We may write

$$
I=\sum_{n \in \mathbb{N}} \sum_{(i, j, k, \ell, r, s) \in S(n)} c(i, j, k, \ell, r, s) A^{i} B^{j} C^{k} D^{\ell} \alpha^{r} \beta^{s} .
$$

Applying $\zeta$ to (5.5) we have

$$
0=\sum_{n \in \mathbb{N}} \sum_{(i, j, k, \ell, r, s) \in S(n)} c(i, j, k, \ell, r, s)\left(A^{\zeta}\right)^{i}\left(B^{\zeta}\right)^{j}\left(C^{\zeta}\right)^{k}\left(D^{\zeta}\right)^{\ell}\left(\alpha^{\zeta}\right)^{r}\left(\beta^{\zeta}\right)^{s} .
$$


Since $I \neq 0$ there exists at least one $n \in \mathbb{N}$ with $S(n) \neq \varnothing$. Set

$$
N=\max \{n \mid S(n) \neq \varnothing\} .
$$

Among the elements in $S(N)$ we choose a 6 -tuple $(i, j, k, \ell, r, s)$ that has the maximum value at $\ell$. In what follows we evaluate the coefficient of

$$
X^{2 i} Y^{2 j} Z^{2 k+\ell} \kappa^{\ell} \lambda^{2 s} \mu^{2 r}
$$

in the right-hand side of (5.6) with respect to the $\mathbb{F}$-basis (3.5) for $\mathfrak{B} \mathfrak{I}$. Denote by $c$ the coefficient. Suppose that $\left(i^{\prime}, j^{\prime}, k^{\prime}, \ell^{\prime}, r^{\prime}, s^{\prime}\right)$ is a 6 -tuple in $S(n)$ for some $n \in \mathbb{N}$ such that

$$
\left(A^{\zeta}\right)^{i^{\prime}}\left(B^{\zeta}\right)^{j^{\prime}}\left(C^{\zeta}\right)^{k^{\prime}}\left(D^{\zeta}\right)^{\ell^{\prime}}\left(\alpha^{\zeta}\right)^{r^{\prime}}\left(\beta^{\zeta}\right)^{s^{\prime}}
$$

contributes to the coefficient $c$. By Theorem 3.4 the monomial (5.7) lies in $\mathfrak{B} \mathfrak{I}_{N}$ not in $\mathfrak{B} \mathfrak{I}_{N-1}$. By Lemma 5.2 the term (5.8) lies in $\mathfrak{B I}_{n}$. It follows from (N2) that $n \geq N$ and the maximality of $N$ implies $n=N$. By Lemma 5.3(i) we have $\ell^{\prime} \geq \ell$ and the maximality of $\ell$ forces that $\ell^{\prime}=\ell$. Combined with Lemma 5.3(ii) this yields that $\left(i^{\prime}, j^{\prime}, k^{\prime}, r^{\prime}, s^{\prime}\right)=(i, j, k, r, s)$. Therefore

$$
\left(A^{\zeta}\right)^{i}\left(B^{\zeta}\right)^{j}\left(C^{\zeta}\right)^{k}\left(D^{\zeta}\right)^{\ell}\left(\alpha^{\zeta}\right)^{r}\left(\beta^{\zeta}\right)^{s}
$$

is the only summand in the right-hand side of (5.6) contributes to the coefficient $c$. By Lemma 5.3(iii) the coefficient $c$ is the nonzero scalar

$$
(-1)^{s} \cdot 4^{-i-j-k-2 \ell-3 r-3 s} \cdot c(i, j, k, \ell, r, s) .
$$

It follows from Theorem 3.4 that the right-hand side of (5.6) is nonzero, a contradiction. The theorem follows.

As a consequence of Theorem 5.4 the $\mathbb{F}$-algebra homomorphism $\natural \circ \zeta: \Re \rightarrow \mathfrak{H}$ described in Section 4 is injective. Note that [25, Theorem 4.5] is a $q$-analogue of the injectivity for $\downarrow \circ \zeta$.

\section{The images of the Casimir elments of $\Re$ under $\zeta$}

In light of Theorem 5.4 the Racah algebra $\Re$ can be viewed as an $\mathbb{F}$-subalgebra of the Bannai-Ito algebra $\mathfrak{B I}$ via $\zeta$.

Lemma 6.1. The element $\iota$ is in the centralizer of $\Re$ in $\mathfrak{B} \mathfrak{I}$.

Proof. By Theorem 4.1 and (3.1) the commutator $[\iota, A]$ is equal to $\frac{1}{4}$ times

$$
\left[Y+Z, X^{2}\right]-[Y+Z, X]
$$

Simplifying (6.1) by using Lemma 4.2(i) yields that (6.1) is zero. Therefore $\iota$ commutes with $A$. Similarly $\iota$ commutes with $B$ and $C$. Combined with Lemma 2.2(i) the lemma follows.

By Lemma 6.1 each of $\iota, \kappa, \lambda, \mu$ lies in the centralizer of $\Re$ in $\mathfrak{B I}$. The intention of the final section is to show that each Casimir element of $\Re$ can be uniquely expressed as a polynomial in $\iota, \kappa, \lambda, \mu$ with coefficients in $\mathbb{F}$.

Throughout this section, let $\left\{\mathfrak{B I}_{n}\right\}_{n \in \mathbb{N}}$ denote the $\mathbb{F}$-subspaces of $\mathfrak{B} \mathfrak{I}$ associated with

$$
\left(w_{X}, w_{Y}, w_{Z}, w_{\kappa}, w_{\lambda}, w_{\mu}\right)=(1,1,2,0,0,0) .
$$

Since the sequence (6.2) satisfies (3.6)-(3.8), it follows from Theorem 3.5 that $\left\{\mathfrak{B}_{n}\right\}_{n \in \mathbb{N}}$ is an $\mathbb{N}$-filtration of $\mathfrak{B} \mathfrak{I}$. 
Lemma 6.2. $Z^{n}=\iota^{n} \bmod \mathfrak{B I}_{2 n-1}$ for all $n \in \mathbb{N}$.

Proof. Proceed by induction on $n$. It is trivial for $n=0$. By (3.1) we have

$$
\iota-Z=X+Y \in \mathfrak{B I}_{1} .
$$

Hence the lemma holds for $n=1$. Suppose that $n \geq 2$. We divide $\iota^{n}-Z^{n}$ into

$$
Z\left(\iota^{n-1}-Z^{n-1}\right)+(\iota-Z) \iota^{n-1} .
$$

Since $Z \in \mathfrak{B I}_{2}$ and by induction hypothesis, the first summand of (6.4) is in $\mathfrak{B I}_{2 n-1}$. By (3.1) the element $\iota \in \mathfrak{B I}_{2}$ and hence $\iota^{n-1} \in \mathfrak{B I}_{2 n-2}$. Combined with (6.3) the second summand of (6.4) is in $\mathfrak{B I}_{2 n-1}$. The lemma follows.

\section{Lemma 6.3.}

(i) For all $n \in \mathbb{N}$ the elements

$$
X^{i} Y^{j} Z^{k} \kappa^{r} \lambda^{s} \mu^{t}+\mathfrak{B I}_{n-1} \quad \text { for all } i, j, k, r, s, t \in \mathbb{N} \text { with } i+j+2 k=n
$$

are an $\mathbb{F}$-basis for $\mathfrak{B I}_{n} / \mathfrak{B I}_{n-1}$.

(ii) For all $n \in \mathbb{N}$ the elements

$$
X^{i} Y^{j} \iota^{k} \kappa^{r} \lambda^{s} \mu^{t}+\mathfrak{B I}_{n-1} \quad \text { for all } i, j, k, r, s, t \in \mathbb{N} \text { with } i+j+2 k=n
$$

are an $\mathbb{F}$-basis for $\mathfrak{B I}_{n} / \mathfrak{B I}_{n-1}$.

(iii) For all $n \in \mathbb{N}$ the elements

$$
X^{i} Y^{j} \iota^{k} \kappa^{r} \lambda^{s} \mu^{t} \quad \text { for all } i, j, k, r, s, t \in \mathbb{N} \text { with } i+j+2 k \leq n
$$

are an $\mathbb{F}$-basis for $\mathfrak{B I}_{n}$.

Proof. (i) Immediate from Theorem 3.4 and the construction of $\left\{\mathfrak{B} \mathfrak{I}_{n}\right\}_{n \in \mathbb{N}}$.

(ii) Immediate from Lemma 6.2 and (i).

(iii) Using (ii) the statement (iii) follows by a routine induction on $n$.

Theorem 6.4. The elements

$$
X^{i} Y^{j} \iota^{k} \kappa^{r} \lambda^{s} \mu^{t} \quad \text { for all } i, j, k, r, s, t \in \mathbb{N}
$$

are an $\mathbb{F}$-basis for $\mathfrak{B I}$.

Proof. Immediate from (N1) and Lemma 6.3(iii).

Corollary 6.5. The elements $\iota, \kappa, \lambda, \mu$ of $\mathfrak{B} \mathfrak{I}$ are algebraically independent over $\mathbb{F}$.

Proof. Immediate from Theorem 6.4.

Lemma 6.6. The $\mathbb{F}$-algebra $\mathfrak{B} \mathfrak{I}$ has a presentation with generators $X, Y, \iota, \kappa, \lambda, \mu$ and relations

$$
\begin{aligned}
& Y X=-X Y-X-Y+\iota+\kappa, \\
& \iota Y=2 Y^{2}-Y \iota-Y+\iota+\kappa+\lambda, \\
& \iota X=2 X^{2}-X \iota-X+\iota+\kappa+\mu, \\
& \kappa X=X \kappa, \quad \kappa Y=Y \kappa, \quad \kappa \iota=\iota \kappa, \quad \lambda=Y \lambda, \quad \lambda \iota=\iota \lambda, \quad \lambda \kappa=\kappa \lambda, \quad \\
& \lambda X=X \lambda, \quad \lambda Y=Y \lambda, \quad \mu \iota=\iota \mu, \quad \mu \kappa=\kappa \mu, \quad \mu \lambda=\lambda \mu . \\
& \mu X=X \mu, \quad \mu Y=Y \mu, \quad \mu \lambda, \quad
\end{aligned}
$$


Proof. This is a reformulation of Proposition 3.3 by using (3.1).

Recall the $D_{6}$-symmetric Casimir elements $\Omega_{A}, \Omega_{B}, \Omega_{C}$ of $\Re$ from (2.16)-(2.18).

Proposition 6.7. The $D_{6}$-symmetric Casimir elements $\Omega_{A}, \Omega_{B}, \Omega_{C}$ of $\Re$ have the following expressions:

$$
\begin{aligned}
& \Omega_{A}=\frac{\lambda(\lambda-2 \iota+3)\left(4 \iota^{2}-8 \iota-4 \kappa-4 \lambda-4 \mu+7\right)}{1024}-\Gamma, \\
& \Omega_{B}=\frac{\mu(\mu-2 \iota+3)\left(4 \iota^{2}-8 \iota-4 \kappa-4 \lambda-4 \mu+7\right)}{1024}-\Gamma, \\
& \Omega_{C}=\frac{\kappa(\kappa-2 \iota+3)\left(4 \iota^{2}-8 \iota-4 \kappa-4 \lambda-4 \mu+7\right)}{1024}-\Gamma,
\end{aligned}
$$

where

$$
\begin{aligned}
\Gamma= & \frac{3(2 \iota+3)(2 \iota+1)(2 \iota-5)(2 \iota-7)}{4096}-\frac{(2 \iota+1)(6 \iota-13)(\kappa+\lambda+\mu)}{512} \\
& +\frac{(\kappa+\lambda+\mu)(\kappa+\lambda+\mu+4)}{64}-\frac{(2 \iota-3)(\kappa \lambda+\lambda \mu+\mu \kappa)}{512}+\frac{\kappa \lambda \mu}{256} .
\end{aligned}
$$

Proof. Applying Theorem 4.1 and Proposition 4.3 to (2.16) and replacing $Z$ by $\iota-X-Y$, we may express $\Omega_{A}$ in terms of $X, Y, \iota, \kappa, \lambda, \mu$. To get (6.6) we apply Lemma 6.6 to express the resulting expression as an $\mathbb{F}$-linear combination of (6.5). Combined with Lemma 2.8 and Proposition 3.2 we obtain (6.7) and (6.8).

Theorem 6.8. For each Casimir element $\Omega$ of $\Re$ there exists a unique four-variable polynomial $P\left(x_{1}, x_{2}, x_{3}, x_{4}\right)$ over $\mathbb{F}$ such that

$$
\Omega=P(\iota, \kappa, \lambda, \mu) .
$$

Proof. By Definition 2.6 there exists a four-variable polynomial $Q\left(y_{1}, y_{2}, y_{3}, y_{4}\right)$ over $\mathbb{F}$ such that $\Omega=\Omega_{A}+Q(\alpha, \beta, \gamma, \delta)$. Set $\widehat{Q}\left(x_{1}, x_{2}, x_{3}, x_{4}\right)=Q\left(y_{1}, y_{2}, y_{3}, y_{4}\right)$ by substituting

$$
\begin{array}{ll}
y_{1}=\frac{\left(2 x_{1}-x_{2}-x_{4}-3\right)\left(x_{2}-x_{4}\right)}{64}, & y_{2}=\frac{\left(2 x_{1}-x_{3}-x_{2}-3\right)\left(x_{3}-x_{2}\right)}{64}, \\
y_{3}=\frac{\left(2 x_{1}-x_{4}-x_{3}-3\right)\left(x_{4}-x_{3}\right)}{64}, & y_{4}=\frac{x_{1}^{2}-2 x_{1}-x_{2}-x_{3}-x_{4}}{4}-\frac{9}{16} .
\end{array}
$$

It follows from Theorem 4.1 that $\Omega=\Omega_{A}+\widehat{Q}(\iota, \kappa, \lambda, \mu)$. Combined with Proposition 6.7 the existence follows. The uniqueness is immediate from Corollary 6.5 .

\section{Acknowledgements}

The research is supported by the Ministry of Science and Technology of Taiwan under the project MOST 106-2628-M-008-001-MY4.

\section{References}

[1] Bergman G.M., The diamond lemma for ring theory, Adv. Math. 29 (1978), 178-218.

[2] Crampé N., Frappat L., Vinet L., Centralizers of the superalgebra osp(1|2): the Brauer algebra as a quotient of the Bannai-Ito algebra, J. Phys. A: Math. Theor. 52 (2019), 424001, 11 pages, arXiv:1906.03936.

[3] De Bie H., De Clercq H., The $q$-Bannai-Ito algebra and multivariate $(-q)$-Racah and Bannai-Ito polynomials, J. London Math. Soc., to appear, arXiv:1902.07883. 
[4] De Bie H., De Clercq H., van de Vijver W., The higher rank q-deformed Bannai-Ito and Askey-Wilson algebra, Comm. Math. Phys. 374 (2020), 277-316, arXiv:1805.06642.

[5] De Bie H., Genest V.X., Tsujimoto S., Vinet L., Zhedanov A., The Bannai-Ito algebra and some applications, J. Phys. Conf. Ser. 597 (2015), 012001, 16 pages, arXiv:1411.3913.

[6] De Bie H., Genest V.X., van de Vijver W., Vinet L., Bannai-Ito algebras and the osp $(1 ; 2)$ superalgebra, in Physical and Mathematical Aspects of Symmetries, Proceedings of the 31st International Colloquium in Group Theoretical Methods in Physics (Rio de Janeiro, June 19-25, 2016), Editors Duarte S., Gazeau J.-P., Faci S., Micklitz T., Scherer R., Toppan F., Springer, Cham, 2017, 349-354, arXiv:1610.04797.

[7] De Bie H., Genest V.X., van de Vijver W., Vinet L., A higher rank Racah algebra and the $\mathbb{Z}_{2}^{n}$ Laplace-Dunkl operator, J. Phys. A: Math. Theor. 51 (2018), 025203, 20 pages, arXiv:1610.02638.

[8] De Bie H., Genest V.X., Vinet L., A Dirac-Dunkl equation on $S^{2}$ and the Bannai-Ito algebra, Comm. Math. Phys. 344 (2016), 447-464, arXiv:1501.03108.

[9] De Bie H., Genest V.X., Vinet L., The $\mathbb{Z}_{2}^{n}$ Dirac-Dunkl operator and a higher rank Bannai-Ito algebra, Adv. Math. 303 (2016), 390-414, arXiv:1511.02177.

[10] Genest V.X., Lapointe L., Vinet L., osp (1,2) and generalized Bannai-Ito algebras, Trans. Amer. Math. Soc. 372 (2019), 4127-4148, arXiv:1705.03761.

[11] Genest V.X., Vinet L., Zhedanov A., The Bannai-Ito algebra and a superintegrable system with reflections on the two-sphere, J. Phys. A: Math. Theor. 47 (2014), 205202, 13 pages, arXiv:1401.1525.

[12] Genest V.X., Vinet L., Zhedanov A., The Bannai-Ito polynomials as Racah coefficients of the $\mathfrak{s l}_{-1}(2)$ algebra, Proc. Amer. Math. Soc. 142 (2014), 1545-1560, arXiv:1205.4215.

[13] Genest V.X., Vinet L., Zhedanov A., The equitable Racah algebra from three $\mathfrak{s u}(1,1)$ algebras, J. Phys. A: Math. Theor. 47 (2014), 025203, 12 pages, arXiv:1309.3540.

[14] Genest V.X., Vinet L., Zhedanov A., The Racah algebra and superintegrable models, J. Phys. Conf. Ser. 512 (2014), 012011, 15 pages, arXiv:1312.3874.

[15] Genest V.X., Vinet L., Zhedanov A., Superintegrability in two dimensions and the Racah-Wilson algebra, Lett. Math. Phys. 104 (2014), 931-952, arXiv:1307.5539.

[16] Genest V.X., Vinet L., Zhedanov A., Embeddings of the Racah algebra into the Bannai-Ito algebra, SIGMA 11 (2015), 050, 11 pages, arXiv:1504.00558.

[17] Genest V.X., Vinet L., Zhedanov A., A Laplace-Dunkl equation on $S^{2}$ and the Bannai-Ito algebra, Comm. Math. Phys. 336 (2015), 243-259, arXiv:1312.6604.

[18] Genest V.X., Vinet L., Zhedanov A., The non-symmetric Wilson polynomials are the Bannai-Ito polynomials, Proc. Amer. Math. Soc. 144 (2016), 5217-5226, arXiv:1507.02995.

[19] Granovskiu Y.A., Zhedanov A.S., Nature of the symmetry group of the $6 j$-symbol, Soviet Phys. JETP 94 (1988), 1982-1985.

[20] Granovskiu Y.I., Zhedanov A.S., Lutsenko I.M., Quadratic algebras and dynamical symmetry of the Schrödinger equation, Soviet Phys. JETP 99 (1991), 205-209.

[21] Huang H.W., Finite-dimensional modules of the Racah algebra and the additive DAHA of type $\left(C_{1}^{\vee}, C_{1}\right)$, arXiv:1906.09160.

[22] Huang H.W., Bockting-Conrad S., The Casimir elements of the Racah algebra, J. Algebra Appl., to appear, arXiv:1711.09574.

[23] Lévy-Leblond J.M., Lévy-Nahas M., Symmetrical coupling of three angular momenta, J. Math. Phys. 6 (1965), 1372-1380.

[24] Terwilliger P., The universal Askey-Wilson algebra, SIGMA 7 (2011), 069, 24 pages, arXiv:1104.2813.

[25] Terwilliger P., The universal Askey-Wilson algebra and DAHA of type $\left(C_{1}^{\vee}, C_{1}\right)$, SIGMA 9 (2013), 047, 40 pages, arXiv:1202.4673.

[26] Tsujimoto S., Vinet L., Zhedanov A., Dunkl shift operators and Bannai-Ito polynomials, Adv. Math. 229 (2012), 2123-2158, arXiv:1106.3512. 